\section{Neuropsychological analyses of impulsiveness in childhood hyperactivity ${ }^{\dagger}$}

\author{
K. RUBIA, E. TAYLOR, A. B. SMITH, H. OKSANNEN, S. OVERMEYER, \\ and S. NEWMAN
}

Impulsiveness is invoked as the key clinical and cognitive problem in children with attention-deficit hyperactivity disorder (ADHD) (Taylor, 1998, 1999; Rubia, 2001) and seems to be responsible for the adverse functional outcome (Taylor et al, 1996). The nature of the pathological alterations in impulse control specific to ADHD, however, needs to be more clearly defined at the behavioural and psychological levels, to improve clinical analysis. Deficits in inhibitory and attentional mechanisms mediated by the frontal lobes have been made responsible for the impulsive features in ADHD, including motor inhibition deficits, prepotent response style, delay aversion, poor protection from interference, problems with cognitive flexibility and timing, social disruption and emotional disinhibition (Barkley, 1997; Rubia, 2001). Here we apply a clinically convenient neuropsychological measure, the Maudsley Attention and Response Suppression (MARS) task battery, designed to measure impulsivity in its different manifestations of motor control, including response inhibition, motor timing and sensorimotor coordination, to children with ADHD, other psychiatric disorders and community controls. Functional magnetic resonance imaging (fMRI) data are presented to elucidate the neuronal correlates of impulsivity.

\section{METHOD}

\section{Experimental study}

\section{Subjects}

Fifty-six children aged 7-15 years participated in the experimental study. All children in the ADHD and psychiatric control groups received a systematic assessment at a research clinic, including full parental and child interviews to yield information for the HYPESCHEME system (Curran et al, 2000). The ADHD group included 16

†See editorial, pp. 93-94, this issue. children with the clinical diagnosis of both ADHD (DSM-IV; American Psychiatric Association, 1994) and hyperkinetic disorder (ICD-10; World Health Organization, 1992, 1993); there were 13 boys and 3 girls, mean age 11.0 (s.d. 2.4) years. They scored above the cut-off point for hyperactive behaviour on parent and teacher versions of the Strengths and Difficulties Questionnaire (SDQ; Goodman, 1997). Four of the children also met the criteria for hyperkinetic conduct disorder, considered to be a subgroup of hyperkinetic disorder (Taylor $e t$ al, 1996); no other comorbid diagnoses were present.

The psychiatric control group consisted of 16 children with psychiatric disorders other than ADHD; there were 12 boys and 4 girls, mean age 11.2 (s.d. 2.3) years. All had been referred to the same clinic because of disruptive behaviour, but did not meet the criteria for hyperkinetic disorder and received an alternative diagnosis: oppositional defiant disorder (number of subjects 2), Asperger syndrome (2), mixed emotional and conduct disorder (2), conduct disorder (2), frontal lobe epilepsy (1), frontal lobe syndrome (1), developmental dyspraxia (2), mild depression (2), Tourette syndrome (1), disorder of developing personality (1) and dysthymia (1). The community control group consisted of 23 children: 19 boys and 4 girls, mean age 10.6 (s.d. 2.3) years, who rated below the threshold level on the parent version of the SDQ.

Subjects were free of medication for at least 48 hours prior to testing. General IQ was assessed using four sub-tests of the Wechsler Intelligence Scale for Children Revised (WISC-R), which has a correlation of 0.93-0.95 with the full administration of the WISC-R (Groth-Marnat, 1990). Children with an IQ lower than 50 were excluded. No group differences were observed in IQ. Written consent was obtained from the parents, and the study was approved by the local ethics committee.

\section{The MARS Task Battery}

Go/nogo task. A motor response has to be selectively executed or inhibited depending on whether a 'go' or 'no go' stimulus appears on a computer screen. The 'go' stimulus, an image of an aeroplane, appeared $70 \%$ of the time; the 'no go' stimulus was an image of a bomb ( $30 \%$ of trials). The task was administered in two blocks of 90 trials, the first block serving as a practice run. The interstimulus interval (ISI) was $1600 \mathrm{~ms}$ : 
the stimulus duration was $200 \mathrm{~ms}$, followed by a blank screen for $1400 \mathrm{~ms}$. Instructions were to respond as fast as possible to the 'go' stimulus (aeroplanes), but not to respond to the 'no go' stimulus (bombs).

Stop task. The 'stop' task requires inhibition of an already planned motor response. A 'go' signal (aeroplane) is converted $a$ posteriori into a 'stop' signal by the appearance of a bomb shortly after the aeroplane. An aeroplane appearing on the screen (ISI $1650 \mathrm{~ms}$ : duration of stimulus $1000 \mathrm{~ms}$, followed $70 \%$ of the time by $650 \mathrm{~ms}$ of blank screen) was followed in $30 \%$ of trials ( $250 \mathrm{~ms}$ later in $20 \%$ of trials and $150 \mathrm{~ms}$ later in $10 \%$ of trials) by an image of a bomb, which replaced the aeroplane for $300 \mathrm{~ms}$, and was then followed by a blank screen for $1100 \mathrm{~ms}$. The children were required to press a button with their right index finger if the aeroplane appeared alone, and not if the aeroplane was followed by the bomb (see Rubia et al, 1998a). Two blocks of 90 trials were administered, starting with a practice block of 90 trials. Both inhibition tasks were administered in randomised order.

Reversal task. In this test of cognitive flexibility a previously learned stimulus-response association ('respond to aeroplanes, not bombs') had to be inhibited in order to learn a new stimulus-response association ('respond to bombs, not aeroplanes'). Subjects were requested to press a response button to bombs appearing now in $70 \%$ of trials, and not to respond to the aeroplanes (30\% of trials) (ISI $1600 \mathrm{~ms}$, stimulus duration $200 \mathrm{~ms}$ ). One block of 90 trials was administered without practice.

Inhibition of synchronised finger-tapping. While 'go/no go' and 'stop' tasks test for inhibition of discrete responses, this task explores the ability to periodically interrupt a continuous movement 'automatic finger-tapping', to wait for several seconds, and then to re-engage in finger-tapping. A 'go' stimulus (an image of Concorde) appeared on the screen (ISI $600 \mathrm{~ms}$ ) on 150 trials. Subjects were instructed to tap regularly in exactly the same rhythm as they saw the plane appearing on the screen. On 15 trials ( $10 \%$ of trials) a 'stop' signal (a black aeroplane) appeared after a random series of 'go' signals, and subjects had to interrupt their tapping as soon as they saw the black aeroplane appear. Each 'stop' signal was followed by a break of $2500 \mathrm{~ms}$, where subjects had to 'hold' their response inhibition period and wait for Concorde to appear again. The break period aimed to investigate possible deficits in hyperactive children to keep a response interruption for a period of several seconds $(2500 \mathrm{~ms})$. The task thus tests for both the ability to interrupt automatic movements and the ability to keep the interruption period for $2500 \mathrm{~ms}$. There was no practice in this task.

Synchronised finger-tapping task. This task tests the ability to synchronise the motor output to a sensory input. Ninety consecutive 'go' signals (Concorde) appeared on the screen (ISI $700 \mathrm{~ms}$ ). Subjects had to synchronise their motor responses as closely and accurately as possible to the onset of stimulus appearance (Rubia et al, 1999b). There was no practice in this task.

Delay task. This tests the ability to synchronise a motor response to a stimulus (aeroplane) which appeared on the screen every $5000 \mathrm{~ms}$ (ISI) with a duration of $200 \mathrm{~ms}$. The task requires time estimation, i.e. the ability to estimate the time interval of $5 \mathrm{~s}$, and motor timing, i.e. the ability to adjust the motor response to this estimated time interval (Rubia et al, 1998b, 1999a). Two blocks of 15 aeroplanes were presented after a practice block of 15 aeroplanes.

\section{Neuroimaging study}

\section{Subjects}

Functional magnetic resonance imaging data were obtained from 16 dextral adolescents on the stop, delay and motor tapping tasks. The data on the stop and delay tasks have been published elsewhere (Rubia et al, $1999 a, 2000)$. Seven clinically referred and unmedicated adolescents with ADHD (age range $12-18$ years; mean age 15.7 , s.d. 2.1 years) were compared with 9 healthy adolescents (age range 12-19 years; mean age 15.01, s.d. 2.3 years), matched for handedness and IQ. Activation tasks were nearly identical to the stop, delay and tapping tasks described in the experimental study design. The stop task, with a probability of stop signals of $50 \%$ and of $30 \mathrm{~s}$ duration, alternated five times with a motor control task (30 s) with identical visual stimulation, requiring $100 \%$ of motor responses (30 s). The delay task (30 s) alternated five times with the motor tapping task (30 s) in a parametric fMRI task design. Both experiments lasted 5 minutes (for more details on the study design, see Rubia et al, 1999a, 2000).

\section{Functional MRI data acquisition and analysis}

After limiting head movements, gradientecho echoplanar MR images were acquired on a 1.5 tesla GE Signa (General Electrics, Milwaukee, USA) system. In each of 14 noncontiguous planes parallel to the anteriorposterior commissure, $100 T_{2}{ }^{*}$-weighted MR images depicting blood oxygen leveldependent (BOLD) contrast were acquired (TE $40 \mathrm{~ms}$, TR $3 \mathrm{~s}$, flip angle $90^{\circ}$, in-plane resolution $3.1 \mathrm{~mm}$, slice thickness $5 \mathrm{~mm}$, slice skip $0.5 \mathrm{~mm}$ ), which were co-registered on a corresponding 43-slice, high-resolution structural echoplanar image.

Functional MRI time series analyses have been described elsewhere in detail (Bullmore et al, 1996; Brammer et al, 1997). The fundamental power quotient (FPQ) of periodic signal change at the frequency of alternation between control and activation conditions was estimated by fitting a sinusoidal regression model using an iterated least squares procedure to the movement-corrected time fMRI series at each single voxel. Generic maps were constructed to represent FPQ and time series at each voxel of each observed data-set by a random permutation procedure and, after smoothing, these were transformed into the standard space of Talairach \& Tournoux (1988). To estimate the difference between comparison and hyperactive groups in the mean power of response to the activation condition, while covarying for IQ, an analysis of covariance (ANCOVA) model was fitted at each voxel generically activated by the activation and the control conditions, respectively, in one or both of the groups using a non-parametric permutation procedure at a probability level of $P<0.05$. There was no significant difference between the two groups on any measure of stimuluscorrelated motion.

\section{RESULTS}

\section{Experimental study}

Multiple one-way analyses of variance were used, with the diagnostic group as a betweensubject factor with three levels (community controls, psychiatric controls and hyperactive children). Post hoc analyses were carried out using least significant difference tests. 


\section{Go/no go task}

No group differences were observed in mean reaction time (MRT). There was a main group effect for variability of responses $(F=4.31$, d.f. $=2.52, P<0.02)$, omission errors $(F=4.25$, d.f. $=2.52, P<0.02)$ and probability of inhibition $(F=3.64$, d.f. $=3$, $P<0.03)$. Post hoc analyses showed that hyperactive children compared with the community group made more variable responses $(P<0.006)$, more omission errors $(P<0.005)$ and had lower probability of inhibition $(P<0.009)$. No differences were found between hyperactive and psychiatric control children or psychiatric control and community groups. There was a negative correlation between the number of omission errors and probability of inhibition $(r=-0.53, P<0.01)$, showing an interdependency of executory and inhibitory processes (Table 1 ).

\section{Stop task}

No group differences were found for the MRT, standard deviation or number of omission errors. However, there was a significant main group effect for impulsive prepotent responses $(F=3.63$, d.f. $=2.52, P<0.03)$ and overall probability of inhibition $(F=3.40$, d.f. $=2.52, P<0.04)$. Hyperactive children compared with community control children showed a higher increase in impulsive prepotent responses, i.e. responding prior to stimulus onset $(P<0.01)$, and showed a lower probability of inhibition $(P<0.001)$. Also in this task there was a negative correlation between number of omission errors and probability of inhibition $(r=-0.27$, $P<0.05)$. There was a trend for hyperactive children to be slower in their stop signal reaction times $(P<0.07)$, a calculated measure of the latency of the stopping process (Schachar \& Logan, 1990) (Table 1).

\section{Reversal task}

There were no group differences in MRT or standard deviation. There was a significant group effect in the number of omission errors $(F=2.89$, d.f. $=2.52, P<0.07)$; hyperactive children committed more omission errors in comparison with both the community control $(P<0.05)$ and the psychiatric control groups $(P=0.05)$, while psychiatric control and community control groups did not differ from one another. There was also a significant group effect for the probability of inhibition $(F=3.3$, d.f. $=2.52, P<0.05)$; hyperactive children showed a significantly lower probability of inhibition compared with the community control children $(P<0.01)$, but not compared with the psychiatric control children. Also in this task there was a negative correlation between the number of omission errors and the probability of inhibition $(r=-0.49, P<0.01)$ (Table 1).

Table I Multivariate comparisons for the six tasks of the Maudsley Attention and Response Suppression task battery by group

\begin{tabular}{|c|c|c|c|c|c|c|c|c|c|c|}
\hline \multirow{2}{*}{$\begin{array}{l}\text { Task } \\
\text { Go/no go }\end{array}$} & \multirow{2}{*}{$\begin{array}{l}\text { Measure } \\
\text { MRT (ms) }\end{array}$} & \multicolumn{2}{|c|}{$\begin{array}{l}\text { Community controls } \\
\qquad \begin{array}{l}(n=23) \\
\text { Mean (s.d.) }\end{array}\end{array}$} & \multicolumn{2}{|c|}{$\begin{array}{l}\text { Psychiatric controls } \\
\qquad(n=16) \\
\text { Mean (s.d.) }\end{array}$} & $\begin{array}{c}\text { ADHD } \\
(n=16) \\
\text { Mean (s.d.) }\end{array}$ & \multicolumn{2}{|c|}{$\begin{array}{c}\text { ANOVA } \\
F \text { (d.f.) }\end{array}$} & \multirow{2}{*}{$\begin{array}{c}P \\
0.62\end{array}$} & \multirow[t]{2}{*}{ Between groups } \\
\hline & & 383 & $(65)$ & 363 & $(48)$ & $365 \quad(98)$ & 0.47 & $(2.52)$ & & \\
\hline & SD & 109 & (26) & 129 & (33) & $145 \quad(33)$ & 4.31 & $(2.52)$ & 0.02 & $A D H D>C$ \\
\hline & Omission errors & 4 & (3) & 7 & (6) & $10 \quad(8)$ & 4.25 & $(2.52)$ & 0.02 & $\mathrm{ADHD}>\mathrm{C}$ \\
\hline & $P_{\mathrm{i}}(\%)$ & 77 & (II) & 70 & $(20)$ & $60 \quad(20)$ & 3.64 & $(2.52)$ & 0.03 & $\mathrm{ADHD}>\mathrm{C}$ \\
\hline \multirow[t]{9}{*}{ Stop } & MRT (ms) & 611 & (93) & 541 & $(166)$ & $590(109)$ & 1.58 & $(2.52)$ & 0.22 & \\
\hline & $\mathrm{SD}$ & 90 & $(47)$ & 184 & $(68)$ & $205 \quad(47)$ & 0.63 & $(2.52)$ & 0.54 & \\
\hline & Omission errors & 3.9 & $(4.7)$ & 4.1 & (3) & $5.3(7.6)$ & 0.35 & $(2.52)$ & 0.71 & \\
\hline & Prepotent responses & 0.7 & $(1.2)$ & 2.1 & (4) & $3.4(3.5)$ & 3.63 & $(2.52)$ & 0.03 & $A D H D>C$ \\
\hline & $P_{i}(\%)$ & & & & & & & & & \\
\hline & overall & 79 & (II) & 7I & (16) & $67 \quad(18)$ & 3.40 & $(2.52)$ & 0.04 & $\mathrm{ADHD}>\mathrm{C}$ \\
\hline & $150 \mathrm{~ms}$ & 88 & (II) & 82 & (I7) & $76 \quad(2 I)$ & 2.64 & $(2.52)$ & 0.08 & \\
\hline & $250 \mathrm{~ms}$ & 75 & $(12)$ & 65 & (16) & $63 \quad(20)$ & 3.23 & $(2.52)$ & 0.04 & $A D H D>C$ \\
\hline & SSRT (ms) & 229 & (38) & 224 & (49) & 271 (II3) & 2.27 & $(2.52)$ & 0.11 & \\
\hline \multirow[t]{4}{*}{ Reversal } & MRT (ms) & 383 & $(67)$ & 361 & (56) & $364 \quad(82)$ & 0.59 & $(2.52)$ & 0.56 & \\
\hline & $\mathrm{SD}$ & 113 & (34) & 116 & $(4 I)$ & $139 \quad(62)$ & 1.74 & $(2.52)$ & 0.19 & \\
\hline & Omission errors & 2 & (3) & 2 & $(2)$ & $5 \quad(5)$ & 2.89 & $(2.52)$ & 0.07 & $A D H D>C ; A D H D>P$ \\
\hline & $P_{\mathrm{i}}(\%)$ & 79 & (13) & 69 & $(22)$ & $65 \quad(20)$ & 3.33 & $(2.52)$ & 0.04 & $\mathrm{ADHD}>\mathrm{C}$ \\
\hline \multirow[t]{4}{*}{ Stop of tapping } & MST (ms) & 149 & (II) & 142 & (23) & 152 (19) & 0.45 & $(2.46)$ & 0.64 & \\
\hline & $\mathrm{SD}$ & 85 & (4) & 82 & $(10)$ & $87 \quad(7)$ & 0.41 & $(2.46)$ & 0.65 & \\
\hline & Omission errors & 35 & (7) & 34 & (4) & $41 \quad(15)$ & 2.1 & $(2.46)$ & 0.12 & \\
\hline & Commission errors & 35 & (5) & 37 & $(10)$ & 38 (II) & 0.9 & $(2.46)$ & 0.40 & \\
\hline \multirow[t]{3}{*}{ Tapping } & $\mathrm{MST}(\mathrm{ms})$ & 164 & (95) & 158 & (96) & $165(105)$ & 0.5 & $(2.45)$ & 0.60 & \\
\hline & $\mathrm{SD}$ & 95 & $(4 I)$ & 96 & (13) & $105 \quad(45)$ & 0.44 & $(2.45)$ & 0.64 & \\
\hline & Omission errors & 12 & (7) & 14 & (8) & II (8) & 0.18 & $(2.45)$ & 0.84 & \\
\hline \multirow[t]{2}{*}{ Delay } & MST (ms) & 384 & $(120)$ & 376 & (I52) & $435(213)$ & 0.65 & $(2.52)$ & 0.53 & \\
\hline & SD & 244 & (163) & 254 & (I52) & $318(211)$ & 0.90 & $(2.52)$ & 0.41 & \\
\hline
\end{tabular}

ADHD, attention-deficit hyperactivity disorder; ANOVA, analysis of variance; C, community controls; MRT, mean reaction time; MST, mean synchronisation time; $P$, psychiatric controls; $P_{\mathrm{i}}$, probability of inhibition; SD, intrasubject standard deviation; SSRT, stop signal reaction time. 


\section{Interruption of motor tapping}

No group differences were observed in mean synchronisation time (MST) (calculated as the absolute mean deviation in time from stimulus onset), intrasubject variability of the tapping time, or the proportion of responses made before versus after stimulus onset. There was a trend for hyperactive children to make more omission errors compared with the community control group $(P<0.06)$ and the psychiatric control group $(P<0.08)$ (Table 1$)$. While there were no overall group differences in total number of commission errors, there were significant group differences in the number of multiple (four or more) commission errors during the break period after the stop signals $(F=3.69$, d.f. $=2.52, P<0.033)$. Hyperactive children made more of these multiple commission mistakes than community control children $(P<0.01)$

\section{Motor tapping task}

No group differences were observed in MST, intrasubject variability of tapping time, or the ratio of responses made before versus after stimulus appearance. While the main group effect for variability of time lengths only approached significance $(F=2.6$, d.f. $=2.45 ; P<0.08)$, there was a trend for hyperactive children to be more variable than the psychiatric control children $(P<0.03)$ but not the community control children (Table 1).

\section{Delay task}

There were no group differences in MST, intrasubject variability, or in the proportion of responses made before $v$. after stimulus onset (Table 1).

\section{Functional MRI study}

\section{Stop task}

There was only a trend for the adolescents with ADHD to be faster in MRT and lower in probability of inhibition. Compared with the hyperactive children, the control group showed significantly increased power of functional response $(P<0.05)$ in the right medial and inferior frontal cortex (Brodmann area (BA) 9/45, 22 voxels), the right mesial frontal cortex (BA 8/32, 10 voxels) and the left caudate nucleus (10 voxels) (Fig. 1). No group differences were observed in performance nor in brain activation during the motor execution task, which served as a control condition to the stop task.

\section{Delay task}

No group differences were observed in MST or intrasubject variability. Analysis of covariance showed increased power of response in controls in right anterior (BA $8 / 32,28$ voxels) and posterior cingulate cortex (BA 31, 10 voxels) (BA 6) (Fig. 1).

\section{Motor tapping task}

Hyperactive adolescents did not differ from control subjects in MST or intrasubject variability. No group differences were observed in frontal brain activations. Differences were observed in the exact location of visual brain activation (Fig. 1).

\section{DISCUSSION}

\section{Specificity of inhibitory deficits}

Testing with the MARS neuropsychological task battery, which indexes the voluntary inhibition of motor responses in different contexts and motor timing processes, has shown that children with a refined phenotype of ADHD, but not psychiatric control children, were impaired in go/no go, stop and reversal tasks, all of which require the inhibition of discrete motor responses.
None of the groups was impaired in the process of interrupting continuous motor responses. Neither group was impaired in the delay task, requiring moderate inhibitory and high motor timing function, nor in the finger-tapping task, requiring simple sensorimotor integration. The impairments in hyperactive children were thus specific to the more demanding inhibition tasks requiring inhibition of discrete motor responses and were not due to generalised impairments in the interruption of automatic activities nor motor timing. However, it is possible that motor timing deficits may appear in other contexts or with different task parameters such as longer time delays.

The hyperactive children did not show deficient executory processes in the motor timing tasks, but showed erratic execution processes in terms of omission errors in all inhibition tasks; executory deficits correlated positively with the inhibitory deficits. This finding is in line with observed performance on both stop and change tasks, where hyperactive children are as impaired in the executory re-engagement phase as they are in the inhibitory processes (for overview see Oosterlaan \& Sergeant, 1998). The difficulty in inhibiting responses may have led to

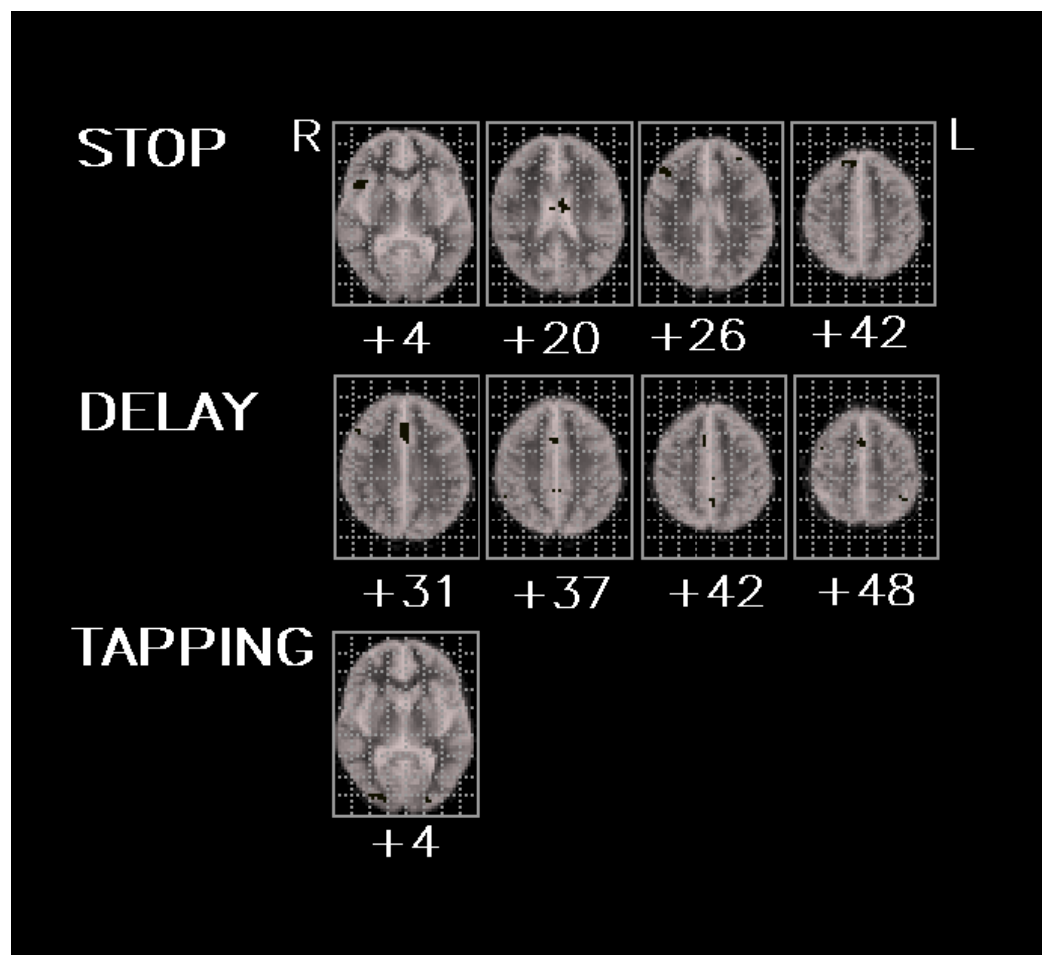

Fig. I Areas of significant difference in power of blood oxygen level-dependent (BOLD) signal response (analysis of covariance map) between seven adolescent boys with attention-deficit hyperactivity disorder $(A D H D)$ and nine without ADHD during performance on the stop, delay and tapping tasks $(P<0.05)$. Black voxels show greater signal response in control subjects. The left side of the image corresponds to the right side of the brain. 
secondary executory deficits. Alternatively, one could assume that the primary difficulty in ADHD is the demand of changing between the two different processes of executory and inhibitory engagements, rather than inhibition alone. This hypothesis would be in line with observed deficits in task-switching in hyperactive children (Reader et al, 1994; Pennington \& Ozonoff, 1996; Kempton et al, 1999) and would point towards a close link between attentional and inhibitory control mechanisms.

\section{No strict diagnostic specificity for inhibitory impairment}

Response inhibition deficits have not been shown to be specific to this one form of psychopathology. While the performance deficits in children with ADHD were specific in relation to norm data (with psychiatric control children being unimpaired), the performance patterns between the psychiatric groups did not differ. Lack of power due to small subject numbers and raised (although sub-threshold) levels of hyperactive behaviours in psychiatric controls may have accounted for the negative findings. Previous studies have shown that anxious children are not impaired in response inhibition tasks, while disruptive children are in some but not all studies (for overview, see Oosterlaan et al, 1998). Further studies using more stringently defined psychiatric control groups will be necessary to investigate the diagnostic specificity of inhibitory impairments.

\section{Specificity of impaired functions}

The findings show that motor timing deficits were not underlying poor performance on the inhibition tasks. However, other underlying deficits in output-related attentional processes or motivation cannot be ruled out. The imaging findings of reduced anterior cingulate activation during both motor timing and response inhibition and its attributed role in response selection and motor attention processes (Frith et al, 1991; Paus et al, 1993; Devinsky et al, 1995) could imply that reduced motor attention and response selection processes have contributed to altered response inhibition; this would be in line with the hypothesis of a deficit in switching between executory and inhibitory processes in ADHD.

A motivational or effort problem is unlikely to account for the inhibitory deficits, since it would have manifested on all tasks. However, problems in motivation may be closely linked to deficit processes and may lead to a positive reinforcement of these impairments via enhanced sensitivity to frustration, resulting in further reduced motivation. Sophisticated cognitive paradigms will need to be designed to address the difficult differentiation of motor-attentional and motivational processes from purely motor inhibitory functions. Our purpose at this stage has been to develop robust measures with face validity for indexing inhibition for the purpose of group discrimination. We are aware that fine distinctions concerning the sub-processes involved, as well as sophisticated context manipulations will be needed in the future to isolate further the specific underlying deficit processes in ADHD.

\section{Brain-behaviour relationships}

The functional imaging data in this study point towards specific right prefrontal impairments during 'frontal lobe' tasks, since the 'non-frontal' activation task (fingertapping) did not elicit underactivation in patients. These right prefrontal deficit findings are in line with other functional imaging studies showing reduced frontostriatal activation in ADHD during go/no go (Vaidya et al, 1998), Stroop (Bush et al, 1999) and attention task performance (Zametkin et al, 1990). The right frontal lobe deficit hypothesis is further supported by structural studies (Castellanos et al, 1996; Casey et al, 1997; Filipek et al, 1997). It may be of interest here that patients with internalising symptoms such as schizophrenia show the inverse pattern of reduced left prefrontal activation during performance on the same stop task (Rubia, 2001; Rubia et al, 2001).

A cross-sectional study comparing healthy adolescents with adults showed that there is a linear increase with age in prefrontal brain activations elicited by stop and delay tasks (Rubia et al, 2000). The hypofrontality in ADHD in these same brain areas thus supports the hypothesis of a prefrontal dysmaturation.

Neuroimaging studies have only just started to be applied to subjects with ADHD to elucidate the neural network substrates of cognitive impairments (Rubia \& Smith, 2001). More refined task designs will be needed to isolate specific sub-processes of task performance at neuropsychological and neuroanatomical levels. Modern fMRI analysis techniques will then potentially be the most powerful tool to investigate neurocognitive deficits in clinical populations. An understanding of the relationship between behaviour, psychology and the brain will ultimately help treatment of the disorder.

\section{ACKNOWLEDGEMENTS}

S.O. and K.R. were supported by European Fellowships from the European Union Programme for the Training and Mobility of Researchers.

\section{REFERENCES}

American Psychiatric Association (1994) Diagnostic and Statistical Manual of Mental Disorders (4th edn) (DSM-IV). Washington, DC: APA.

Barkley, R. A. (1997) Behavioral inhibition, sustained attention, and executive functions: constructing a unifying theory of ADHD. Psychological Bulletin, I2I, 65-94.

Brammer, M. J., Bullmore, E.T. \& Simmons, A. (1997) Generic brain activation mapping in functional magnetic resonance imaging: a non-parametric approach. Magnetic Resonance Imaging, I5, 763-770.

Bullmore, E.T., Brammer, M. J., Williams, S. C. R., et al (1996) Statistical methods of estimation and inference for functional MR image analysis. Magnetic Resonance in Medicine, 35, 26I-277.

Bush, G., Frazier, J. A., Rauch, S. L., et al (1999) Anterior cingulate cortex dysfunction in attention deficit/hyperactivity disorder revealed by fMRI and the Counting Stroop. Biological Psychiatry, 45, 1542-1552.

Casey, B. J., Trainor, R., Orendi, J. L., et al (1997) A developmental functional MRI study of prefrontal activation during performance of a go-no-go task. Journal of Cognitive Neuroscience, 9, 835-847.

Castellanos, F. X., Giedd, J. N., Marsh, W. L., et al (1996) Quantitative brain magnetic resonance imaging in attention-deficit hyperactivity disorder. Archives of General Psychiatry, 53, 607-616.

Curran, S., Taylor, E. \& Asherson, P. (2000)

HYPESCHEME: an operational criteria checklist and minimum dataset for molecular genetic studies of attention deficit and hyperactivity disorder. Neuropsychiatric Genetics, 96, 244-250.

Devinsky, O., Morrell, M. J. \& Vogt, B. A. (1995) Contributions of the anterior cingulate cortex to behaviour. Brain, II8, 279-306.

Filipek, P. A., Semrud-Clikeman, M., Steingard, R. J., et al (1997) Volumetric MRI analysis comparing subjects having attention-deficit hyperactivity disorder with normal controls. Neurology, 48, 589-60I.

Frith, C. D., Friston, K., Liddle, P. F., et al (199I) Willed action and the prefrontal cortex in man: a study with PET. Proceedings of the Royal Society of London (Biological Sciences), 244, 24I-246.

Goodman, R. (1997) The Strengths and Difficulties Questionnaire: a research note. Journal of Child Psychology and Psychiatry and Allied Disciplines, 38, $581-586$

Groth-Marnat, G. (1990) The Wechsler intelligence scales. In Handbook of Psychological Assessment (2nd edn). New York: John Wiley \& Sons.

Kempton, S., Vance, A., Maruff, E., et al (1999) Executive function and attention deficit hyperactivity disorder: stimulant medication and better executive 
function performance in children. Psychological Medicine, 29, 527-538.

Oosterlaan, J. \& Sergeant, J. A. (1998) Response inhibition and response re-engagement in attentiondeficit/hyperactivity disorder, disruptive, anxious and normal children. Behavioural Brain Research, 94, 33-43.

_ , Logan, G. D. \& Sergeant, J. A. (1998) Response inhibition in $A D / H D, C D$, comorbid $A D / H D+C D$, anxious, and control children: a meta-analysis of studies with the stop task. Journal of Child Psychology and Psychiatry and Allied Disciplines, 39, 4II-425.

Paus, T., Petrides, M., Evans, A. C., et al (1993) Role of the human cingulate cortex in the control of oculomotor, manual, and speech responses: a positron emission tomography study. Journal of Neurophysiology, 70, 453-469.

Pennington, B. F. \& Ozonoff, S. (1996) Executive functions and developmental psychopathology. Journal of Child Psychology and Psychiatry and Allied Disciplines, 37, 51-87.

Reader, M. J., Harris, E. L., Schuerholz, L. J., et al (1994) Attention deficit hyperactivity disorder and executive dysfunction. Developmental Neuropsychology, 10, 493-512.

Rubia, K. (200I) The dynamic approach to ADHD: use of $\mathrm{fMRI}$ combined with neuropsychology to elucidate the dynamics of psychiatric disorders, exemplified in ADHD and schizophrenia. Reviews of Neuroscience, in press.

— \& Smith, A. (200I) Attention-deficit hyperactivity disorder: current findings and treatment. Current Opinion in Psychiatry, $\mathbf{4}$, in press.

\section{_ , Oosterlaan, J., Sergeant, J. A., et al (1998a)}

Inhibitory dysfunction in hyperactive boys. Behavioural Brain Research, 94, 25-32.

_ , Overmeyer, S. O., Taylor, E., et al (1998b)

Prefrontal involvement in temporal bridging and timing movement: an fMRI study. Neuropsychologia, $\mathbf{3 6}$, 1283-1293.

_, _, _, et al (1999a) Hypofrontality in Attention Deficit Hyperactivity Disorder (ADHD) during higher-order motor control: a study using $\mathrm{fMRI}$. American Journal of Psychiatry, 156, 891-896.

\section{_ , Sergeant, J. A., Taylor, E., et al (1999b)}

Synchronization, anticipation and consistency of motor timing in dimensionally defined children with Attention Deficit Hyperactivity Disorder. Perceptual and Motor Skills, 89, 1237-1258.

\section{—, Overmeyer, S., Taylor, E., et al (2000)}

Frontalisation with age: mapping neurodevelopmental trajectories with fMRI. Neuroscience Biobehavioural Review, 24, 13-19.

_ , Russel, T., Bullmore, E., et al (200I) Reduced frontal activation in schizophrenia during normal inhibitory function. Schizophrenia Research, in press.

Schachar, R. \& Logan, G. D. (1990) Impulsivity and inhibitory control in normal development and childhood psychopathology. Developmental Psychology, 26. $710-720$

Swanson, J. M., Sergeant, J. A., Taylor, E., et al (1998) Attention-deficit hyperactivity disorder and hyperkinetic disorder. Lancet, 35I, 429-433.

Talairach, J. \& Tournoux, P. (1988) Co-planar Stereotaxic Atlas of the Human Brain. New York: Thieme Medical.

\section{CLINICAL IMPLICATIONS}

- Development of task batteries that give objective measurement of the cognitive aspects of impulsivity and attentional control will aid clinical assessment of attention-deficit hyperactivity disorder (ADHD) and clarify the close relationship between cognitive and behavioural expressions of impulsivity, facilitating cognitive-behavioural intervention.

- Defining the specificity of the inattentional and inhibitory deficits of children with ADHD by comparisons with other neurodevelopmental psychiatric patient groups will help to narrow the cognitive definition of the disorder.

- Definition of the neural substrates of these cognitive dysfunctions by using refined neuropsychological tasks combined with functional and structural neuroimaging will further the development of new and better targeted biological intervention.

\section{LIMITATIONS}

- The raised sub-threshold levels of hyperactive behaviour in the psychiatric control group may have reduced the effect sizes.

- Future studies are needed to elucidate the specificity of the inhibitory and attentional deficits of ADHD by comparisons with other, more stringently defined, neurodevelopmental psychiatric disorders such as obsessive-compulsive disorder, autism and childhood depression.

- The cognitive subprocesses underlying poor performance on inhibitory and attentional tasks in ADHD could be further isolated by sophisticated task designs, targeting additional potential aspects such as motivation or effort employment.

K. RUBIA, PhD, E. TAYLOR, FRCPsych, A. B. SMITH, PhD, H. OKSANNEN, MSc, S. OVERMEYER, MD, S. NEWMAN, BSc, MRC Child and Adolescent Psychiatry Unit, Institute of Psychiatry, King's College, London

Correspondence: Dr Katya Rubia, MRC Child and Adolescent Psychiatry Unit, Institute of Psychiatry, De Crespigny Park, London SE5 8AF, UK. Tel: +44(0)20 7848 0463; fax: +44(0)20 7705 5800; e-mail: k.rubia@iop.kcl.ac.uk

(First received 24 March 2000, final revision 18 January 200I, accepted I8 January 200I)

Taylor, E. (1998) Clinical foundations of hyperactivity research. Behavioural Brain Research, 94, II-24.

- (1999) Developmental neuropsychopathology of attention deficit and impulsiveness. Development and Psychopathology, II, 607-628.

\section{_ , Chadwick, O., Heptinstall, E., et al (1996)} Hyperactivity and conduct problems as risk factors for adolescent development. Journal of the American Academy of Child and Adolescent Psychiatry, 35, 1213-1226.

Vaidya, C. J., Austin, G., Kirkorian, G., et al (1998) Selective effects of methylphenidate in attention deficit hyperactivity disorder: a functional magnetic resonance study. Proceedings of the National Academy of Sciences of the USA, 95, 14494-14499.
World Health Organization (1993) The ICD-10 Classification of Mental and Behavioural Disorders: Clinical Descriptions and Diagnostic Guidelines. Geneva: WHO.

- (1993) The ICD-10 Classification of Mental and Behavioural disorders: Diagnostic Criteria for Research. Geneva: WHO.

Zametkin, A. J., Nordahl, T. E., Gross, M., et al (1990) Cerebral glucose metabolism in adults with hyperactivity of childhood onset. New England journal of Medicine, 323, 1361-1366. 